\title{
Robustness analysis of global exponential stability of nonlinear stochastic systems with respect to neutral terms and time-varying delays
}

\author{
Zhiying $\mathrm{Xu}^{1}$, Wei Liu², Yan $\mathrm{Li}^{3}$ and Junhao $\mathrm{Hu}^{1 *}$
}

"Correspondence:

junhaohu74@163.com

${ }^{1}$ College of Mathematics and

Statistics, South Central University

for Nationalities, Wuhan, 430074,

P.R. China

Full list of author information is

available at the end of the article

\begin{abstract}
In this paper, we consider a class of nonlinear stochastic systems with respect to neutral terms and time-varying delays. Given a globally exponentially stable nonlinear stochastic system, the robustness of the global exponential stability of the system subject to a time delay and a neutral term can be derived by a subtle inequality and a transcendental equation. The upper bound of the allowable time delays and the neutral terms contraction coefficient is easy to verify and implement. Finally, an example with a numerical simulation is given to illustrate the presented criteria.
\end{abstract}

Keywords: robustness analysis; nonlinear stochastic systems; mean square exponential stability; transcendental equation

\section{Introduction}

In the recent few decades, nonlinear stochastic systems have been widely studied by many authors due to the fact that nonlinear stochastic systems can be applied to population ecology, steam processes, heat exchanges, the distributed networks containing lossless transmission lines, and other engineering systems. Many stochastic systems not only depend on present and past states but also involve derivatives with delays. Neutral stochastic differential delay equations (NSDDEs) are often used to describe such systems. One of the important issues in the study of NSDDEs is the automatic control, with consequent emphasis being placed on the analysis of stability. Kolmanovskii and Nosov [1] not only established the existence and uniqueness of the solution of NSDDEs but also investigated the stability and asymptotic stability of NSDDEs. Mao [2] studied the exponential stability of NSDDEs. Taking the abrupt changes in the structure and parameters of the systems into account, Kolmanovskii et al. [3] considered the moment asymptotic boundedness and moment exponential stability of the solution of NSDDEs with Markovian switching. Mao et al. [4] investigated the almost surely asymptotic stability of NSDDEs. Bao et al. [5] discussed the stability in distribution of NSDDEs with Markovian switching.

On the other hand, the noise and time delays are often the sources of instability and they may destabilize stable systems if they exceed their limits (see $[6,7]$ and $[8])$. Shen

\section{Springer}

( 2015 Xu et al.: licensee Springer. This is an Open Access article distributed under the terms of the Creative Commons Attribution License (http://creativecommons.org/licenses/by/4.0), which permits unrestricted use, distribution, and reproduction in any medium, provided the original work is properly credited. 
and Wang [9] studied the noise-induced stabilization of the recurrent neural networks with mixed time-varying delays and Markovian switching parameters. In [10], they continued to analysis the robustness of global exponential stability of recurrent neural networks in the presence of time delays and random disturbances. In [11-14], the authors investigated the robustness of global exponential stability of stochastic systems (with Markovian switching) in the presence of time-varying delays or noises. The stability of the systems often also depends on a neutral term. Shen and Wang [15] discussed the robustness of global exponential stability of nonlinear systems in the presence of time delays and neutral terms.

In this paper, we will consider a class of nonlinear stochastic systems with respect to neutral terms and time-varying delays. By using a subtle inequality, a sufficient condition ensuring robust exponential stability is obtained and the upper bounds of the allowable time delay and the neutral term contraction coefficient for global exponential stability are characterized. We prove theoretically that, for a globally exponentially stable nonlinear stochastic system, if the time delay and neutral term contraction coefficient are smaller than the derived upper bounds, then the perturbed nonlinear stochastic system is guaranteed to stay globally exponentially stable.

The rest of this paper is organized as follows. Some preliminaries and assumptions are introduced in Section 2. In Section 3, the robustness of global exponential stability of nonlinear stochastic systems with respect to neutral terms and time-varying delays is analyzed and a corollary for our theorem is derived. In Section 4, a numerical example is given to illustrate the theoretical result. Finally, concluding remarks are given in Section 5.

\section{Preliminaries and assumptions}

Throughout this paper, unless otherwise specified, let $\left(\Omega, \mathcal{F},\left\{\mathcal{F}_{t}\right\}_{t \geq 0}, \mathbb{P}\right)$ be a complete probability space with a filtration $\left\{\mathcal{F}_{t}\right\}_{t \geq 0}$ satisfying the usual conditions (i.e., it is right continuous and $\mathcal{F}_{0}$ contains all $\mathbb{P}$-null sets). Let $w(t)=\left(w_{1}(t), \ldots, w_{m}(t)\right)^{T}, t \geq 0$, be an $m$-dimension Brownian motion defined on the probability space. Let $|\cdot|$ be the Euclidean norm in $\mathbb{R}^{n}$. If $A$ is a vector or matrix, its transpose is denoted by $A^{T}$. If $A$ is a matrix, its trace norm is denoted by $|A|=\sqrt{\operatorname{trace}\left(A^{T} A\right)}$. Let $C\left([-\bar{\tau}, 0] ; \mathbb{R}^{n}\right)$ denote the family of continuous functions $\varphi$ from $[-\bar{\tau}, 0]$ to $\mathbb{R}^{n}$ with the norm $\|\varphi\|=\sup _{-\bar{\tau} \leq \theta \leq 0}|\varphi(\theta)|$. For $p>0$, denote by $\mathcal{L}_{\mathcal{F}_{0}}^{p}\left([-\bar{\tau}, 0] ; \mathbb{R}^{n}\right)$ the family of all $\mathcal{F}_{0}$-measurable, $C\left([-\bar{\tau}, 0] ; \mathbb{R}^{n}\right)$-valued random variables $\xi$ such that $\mathbb{E}\|\xi\|^{p}<\infty$. Denote by $\mathcal{C}_{\mathcal{F}_{0}}^{b}\left([-\bar{\tau}, 0], \mathbb{R}^{n}\right)$ the family of all $\mathcal{F}_{0}$-measurable, bounded, and $C\left([-\bar{\tau}, 0] ; \mathbb{R}^{n}\right)$-valued random variables.

In this paper, we will consider a nonlinear neutral type time-varying delay stochastic system of the form

$$
\left\{\begin{array}{l}
\mathrm{d}[y(t)-G(y(t-\tau(t)))] \\
\quad=f(y(t), y(t-\tau(t)), t) \mathrm{d} t+g(y(t), y(t-\tau(t)), t) \mathrm{d} w(t), \quad t>t_{0}, \\
y(t)=\psi\left(t-t_{0}\right), \quad t_{0}-\bar{\tau} \leq t \leq t_{0},
\end{array}\right.
$$

where $f: \mathbb{R}^{n} \times \mathbb{R}^{n} \times \mathbb{R}_{+} \rightarrow \mathbb{R}^{n}, g: \mathbb{R}^{n} \times \mathbb{R}^{n} \times \mathbb{R}_{+} \rightarrow \mathbb{R}^{n \times m}, G: \mathbb{R}^{n} \rightarrow \mathbb{R}^{n}, t_{0}>0, w(t)$ is an $m$-dimension Brownian motion defined on the probability space $\left(\Omega, \mathcal{F},\left\{\mathcal{F}_{t}\right\}_{t \geq 0}, \mathbb{P}\right), \tau(t)$ is a time-varying delay, which satisfies $\tau(t):\left[t_{0},+\infty\right) \rightarrow[0, \bar{\tau}], \dot{\tau}(t) \leq \mu<1, \psi=\{\psi(s):-\bar{\tau} \leq$ $s \leq 0\} \in \mathcal{C}_{\mathcal{F}_{0}}^{b}\left([-\bar{\tau}, 0], \mathbb{R}^{n}\right)$. Assume that $f, g$, and $G$ satisfy the following assumptions: 
Assumption 2.1 For all $u, v, \bar{u}, \bar{v} \in \mathbb{R}^{n}$ and $t \in \mathbb{R}_{+}$, there exists a constant $K_{1}>0$ such that

$$
|f(u, \bar{u}, t)-f(v, \bar{v}, t)| \leq K_{1}(|u-v|+|\bar{u}-\bar{v}|) .
$$

Moreover, for any $t \in \mathbb{R}_{+}, f(0,0, t) \equiv 0$.

Assumption 2.2 For all $u, v, \bar{u}, \bar{v} \in \mathbb{R}^{n}$ and $t \in \mathbb{R}_{+}$, there exists a positive constant $K_{2}$ such that

$$
|g(u, \bar{u}, t)-g(v, \bar{v}, t)| \leq K_{2}(|u-v|+|\bar{u}-\bar{v}|) .
$$

Moreover, for any $t \in \mathbb{R}_{+}, g(0,0, t) \equiv 0$.

Assumption 2.3 For all $u, v \in \mathbb{R}^{n}$, there exists a constant $k \in(0,1)$ such that

$$
|G(u)-G(v)| \leq k|u-v| .
$$

Moreover, $G(0)=0$.

It is well known that for any given initial value $t_{0}$ and $\psi$, according to Assumptions 2.12.3, system (2.1) has a unique state $y\left(t ; t_{0}, \psi\right)$ when $t \geq t_{0}-\bar{\tau}$ [2]. Besides, system (2.1) has a trivial state $y \equiv 0$.

In case of no any time delay and neutral term, system (2.1) has the following form:

$$
\left\{\begin{array}{l}
\mathrm{d} x(t)=f(x(t), x(t), t) \mathrm{d} t+g(x(t), x(t), t) \mathrm{d} w(t), \quad t>t_{0}, \\
x\left(t_{0}\right)=\psi(0) \in \mathbb{R}^{n}
\end{array}\right.
$$

From [2], according to Assumptions 2.1 and 2.2, for any given initial value $t_{0}$ and $\psi(0)$, system (2.2) exists a unique state $x\left(t ; t_{0}, \psi(0)\right)$. And, $x \equiv 0$ is the trivial state of system (2.2). With the purpose of analyzing the stability of systems (2.2) and (2.1), we give the definition of the global exponential stability of the two systems as follows:

Definition 2.1 Let $p \geq 2$, if for any $t_{0} \in \mathbb{R}_{+}$and $\psi(0) \in \mathbb{R}^{n}$, there exist positive constants $\alpha$ and $\beta$ such that

$$
\mathbb{E}\left|x\left(t ; t_{0}, \psi(0)\right)\right|^{p} \leq \alpha|\psi(0)| \exp \left(-\beta\left(t-t_{0}\right)\right), \quad t \geq t_{0},
$$

where $x\left(t ; t_{0}, \psi(0)\right)$ is the state of system (2.2), then the state of system (2.2) is $p$ th moment globally exponentially stable. The state of system (2.1) is $p$ th moment globally exponentially stable if for any $t_{0} \in \mathbb{R}_{+}, \psi \in \mathcal{C}_{\mathcal{F}_{0}}^{b}\left([-\tau, 0] ; \mathbb{R}^{n}\right)$, there exist $\tilde{\alpha}>0$ and $\tilde{\beta}>0$ such that

$$
\mathbb{E}\left|y\left(t ; t_{0}, \psi\right)\right|^{p} \leq \tilde{\alpha}\|\psi\| \exp \left(-\tilde{\beta}\left(t-t_{0}\right)\right), \quad t \geq t_{0},
$$

i.e., the Lyapunov exponent

$$
\limsup _{t \rightarrow \infty}\left(\ln \left(\mathbb{E}\left|y\left(t ; t_{0}, \psi\right)\right|^{p}\right) / t\right)<0,
$$

where $y\left(t ; t_{0}, \psi\right)$ is the state of system (2.1). 
From the above definitions, it is clear that the almost surely global exponential stability of system (2.1) implies the $p$ th moment global exponential stability of system (2.1) (see [2]) but not vice versa. However, if Assumptions 2.1-2.3 hold, we can get the following lemma (see Theorem 4.2, p.128 in [2]).

Lemma 2.1 Let Assumptions 2.1-2.3 hold. The pth moment global exponential stability of system (2.1) implies the almost surely global exponential stability.

Meanwhile, in order to obtain our result, we also need another lemma (see Theorem 7.1, p.39 in [2]).

Lemma 2.2 Let $p \geq 2$ and $h: \mathbb{R}^{n} \times \mathbb{R}^{n} \times\left[t_{0}, t\right] \rightarrow \mathbb{R}^{n \times m}$, such that

$$
\mathbb{E} \int_{t_{0}}^{t}|h(s)|^{p} \mathrm{~d} s<\infty
$$

Then

$$
\mathbb{E}\left|\int_{t_{0}}^{t} h(s) \mathrm{d} w(s)\right|^{p} \leq\left(\frac{p(p-1)}{2}\right)^{p / 2}\left(t-t_{0}\right)^{\frac{p-2}{2}} \mathbb{E} \int_{t_{0}}^{t}|h(s)|^{p} \mathrm{~d} s .
$$

In particular, for $p=2$, there is equality.

\section{Main results}

In this section, we will give the quantitative analysis regarding the effect of time delay and neutral term on global exponential stability of nonlinear stochastic systems. In the following theorem, we will show that if system (2.2) is $p$ th moment globally exponentially stable, system (2.1) may remain to be $p$ th moment globally exponentially stable provided that time delay $\bar{\tau}$ and contraction coefficient $k$ are sufficiently small.

Theorem 3.1 Let Assumptions 2.1-2.3 hold and system (2.2) be pth moment globally exponentially stable, then system (2.1) is pth moment globally exponentially stable and almost surely globally exponentially stable, if $\bar{\tau}<\tilde{\tau}, \tilde{\tau}$ is a unique positive solution of the transcendental equation

$$
\begin{aligned}
& {\left[2 c_{1} \Delta 12^{p-1}(2 k)^{p}\left(\frac{1}{1-\mu}+\frac{1}{1-2 \mu}\right)+2 \frac{4^{p-1} k^{p}}{1-\mu}+4^{p-1} c_{1} c_{2}\right.} \\
& \left.\quad+\frac{c_{1} c_{3}}{8 p \beta}(8 \alpha)^{P}+\left(8^{p-1} c_{1} \bar{\tau}+4^{p-1} k^{p}\right)\left(1+\frac{1}{1-\mu}\right)\right] \\
& \quad \times 2^{p-1} \exp \left(2 \Delta c_{1}\left(2^{p-1}+4^{p-1}\right)+2 \Delta c_{1} c_{3} 8^{p-1}\right)+2^{p-1} \alpha^{p} \exp (-p \beta(\Delta-\bar{\tau}))=1,
\end{aligned}
$$

where

$$
\begin{aligned}
& c_{1}=\left(\frac{4 \Delta}{\varepsilon}\right)^{p-1} K_{1}^{p}+[4 \Delta p(p-1)]^{p / 2} \frac{K_{2}^{p}}{4 \Delta(1-\varepsilon)^{p-1}} \\
& c_{2}=6^{p-1} k^{p} \bar{\tau}\left(\frac{1}{1-\mu}+\frac{2}{1-2 \mu}\right)+\left[(6 \bar{\tau})^{p-1} K_{1}^{p}+\frac{1}{6 \bar{\tau}}[2 p(p-1) \bar{\tau}]^{p / 2}\left(3 K_{2}\right)^{p}\right] \frac{\bar{\tau}^{2}}{1-\mu},
\end{aligned}
$$




$$
c_{3}=\left[(6 \bar{\tau})^{p-1} K_{1}^{p}+\frac{1}{6 \bar{\tau}}[2 p(p-1) \bar{\tau}]^{p / 2}\left(3 K_{2}\right)^{p}\right] \bar{\tau}\left(1+\frac{1}{1-\mu}\right)
$$

$\varepsilon$ is an adjustable parameter, $\varepsilon \in(0,1)$, and $\Delta$ is a step, $\Delta-\bar{\tau}>\ln \left(2^{p-1} \alpha^{p}\right) / p \beta>0$.

Proof Fix $t_{0}, \psi=\{\psi(t),-\bar{\tau} \leq t \leq 0\}$, for simplicity, we write $x\left(t ; t_{0}, \psi(0)\right), y\left(t ; t_{0}, \psi\right)$ as $x(t)$, $y(t)$ respectively. From system (2.1) and system (2.2), for any $t \geq t_{0}$, we have

$$
\begin{aligned}
x(t) & -y(t)+G(y(t-\tau(t)))-G\left(y\left(t_{0}-\tau\left(t_{0}\right)\right)\right) \\
= & \int_{t_{0}}^{t}[f(x(s), x(s), s)-f(y(s), y(s-\tau(s)), s)] \mathrm{d} s \\
& \quad+\int_{t_{0}}^{t}[g(x(s), x(s), s)-g(y(s), y(s-\tau(s)), s)] \mathrm{d} w(s),
\end{aligned}
$$

when $t \leq t_{0}+2 \Delta$, by Assumptions 2.1 and 2.2, the Hölder inequality, and Lemma 2.2, we derive

$$
\begin{aligned}
\mathbb{E} \mid x(t) & -y(t)+G(y(t-\tau(t)))-\left.G\left(y\left(t_{0}-\tau\left(t_{0}\right)\right)\right)\right|^{p} \\
\leq & \frac{1}{\varepsilon^{p-1}} \mathbb{E}\left|\int_{t_{0}}^{t}[f(x(s), x(s), s)-f(y(s), y(s-\tau(s)), s)] \mathrm{d} s\right|^{p} \\
& +\frac{1}{(1-\varepsilon)^{p-1}} \mathbb{E}\left|y \int_{t_{0}}^{t}[g(x(s), x(s), s)-g(y(s), y(s-\tau(s)), s)] \mathrm{d} w(s)\right|^{p} \\
\leq & \left(\frac{2 \Delta}{\varepsilon}\right)^{p-1} \int_{t_{0}}^{t} \mathbb{E}|f(x(s), x(s), s)-f(y(s), y(s-\tau(s)), s)|^{p} \mathrm{~d} s \\
& +\frac{1}{(1-\varepsilon)^{p-1}}[p(p-1) / 2]^{p / 2}(2 \Delta)^{\frac{p-2}{2}} \\
& \times \int_{t_{0}}^{t} \mathbb{E}|g(x(s), x(s), s)-g(y(s), y(s-\tau(s)), s)|^{p} \mathrm{~d} s \\
\leq & \left(\frac{4 \Delta}{\varepsilon}\right)^{p-1} K_{1}^{p}\left[\int_{t_{0}}^{t} \mathbb{E}|x(s)-y(s)|^{p} \mathrm{~d} s+\int_{t_{0}}^{t} \mathbb{E}|x(s)-y(s-\tau(s))|^{p} \mathrm{~d} s\right] \\
& +\frac{1}{4 \Delta}[4 \Delta p(p-1)]^{p / 2} \frac{K_{2}^{p}}{(1-\varepsilon)^{p-1}} \\
& \times\left[\int_{t_{0}}^{t} \mathbb{E}|x(s)-y(s)|^{p} \mathrm{~d} s+\int_{t_{0}}^{t} \mathbb{E}|x(s)-y(s-\tau(s))|^{p} \mathrm{~d} s\right] \\
:= & c_{1} \int_{t_{0}}^{t} \mathbb{E}|x(s)-y(s)|^{p} \mathrm{~d} s+c_{1} \int_{t_{0}}^{t} \mathbb{E}|x(s)-y(s)+y(s)-y(s-\tau(s))|^{p} \mathrm{~d} s \\
\leq & \left(2^{p-1}+1\right) c_{1} \int_{t_{0}}^{t} \mathbb{E}|x(s)-y(s)|^{p} \mathrm{~d} s+2^{p-1} c_{1} \int_{t_{0}}^{t} \mathbb{E}|y(s)-y(s-\tau(s))|^{p} \mathrm{~d} s
\end{aligned}
$$

where $c_{1}=\left(\frac{4 \Delta}{\varepsilon}\right)^{p-1} K_{1}^{p}+\frac{1}{4 \Delta}[4 \Delta p(p-1)]^{p / 2} \frac{K_{2}^{p}}{(1-\varepsilon)^{p-1}}$.

By (2.1) and Assumptions 2.1-2.3, we get

$$
\begin{aligned}
& \mathbb{E}|y(s)-y(s-\tau(s))|^{p} \\
& \quad \leq 3^{p-1} \mathbb{E}|G(y(s-\tau(s)))-G(y(s-2 \tau(s)))|^{p}+3^{p-1} \int_{s-\bar{\tau}}^{s} \mathbb{E}|f(y(r), y(r-\tau(r)), r) \mathrm{d} r|^{p}
\end{aligned}
$$




$$
\begin{aligned}
& +3^{p-1} \mathbb{E}\left|\int_{s-\bar{\tau}}^{s} g(y(r), y(r-\tau(r)), r) \mathrm{d} w(r)\right|^{p} \\
\leq & 3^{p-1} k^{p} \mathbb{E}|y(s-\tau(s))-y(s-2 \tau(s))|^{p} \\
& +(3 \bar{\tau})^{p-1} \int_{s-\bar{\tau}}^{s} \mathbb{E}|f(y(r), y(r-\tau(r)), r)|^{p} \mathrm{~d} r \\
& +3^{p-1}[p(p-1) / 2]^{p / 2}(\bar{\tau})^{\frac{p-2}{2}} \mathbb{E} \int_{s-\bar{\tau}}^{s}|g(y(r), y(r-\tau(r)), r)|^{p} \mathrm{~d} r \\
\leq & 3^{p-1} k^{p} \mathbb{E}|y(s-\tau(s))-y(s-2 \tau(s))|^{p} \\
& +(6 \bar{\tau})^{p-1} K_{1}^{p}\left[\int_{s-\bar{\tau}}^{s} \mathbb{E}|y(r)|^{p} \mathrm{~d} r+\int_{s-\bar{\tau}}^{s} \mathbb{E}|y(r-\tau(r))|^{p} \mathrm{~d} r\right] \\
& +\frac{1}{6 \bar{\tau}}[2 \bar{\tau} p(p-1)]^{p / 2}\left(3 K_{2}\right)^{p}\left[\int_{s-\bar{\tau}}^{s} \mathbb{E}|y(r)|^{p} \mathrm{~d} r+\int_{s-\bar{\tau}}^{s} \mathbb{E}|y(r-\tau(r))|^{p} \mathrm{~d} r\right] .
\end{aligned}
$$

In addition, for $t_{0}+\tau \leq t \leq t_{0}+2 \Delta$,

$$
\begin{aligned}
& 3^{p-1} k^{p} \int_{t_{0}+\bar{\tau}}^{t} \mathbb{E}|y(s-\tau(s))-y(s-2 \tau(s))|^{p} \mathrm{~d} s \\
& \leq 6^{p-1} k^{p} \int_{t_{0}+\bar{\tau}}^{t} \mathbb{E}|y(s-\tau(s))|^{p} \mathrm{~d} s+6^{p-1} k^{p} \int_{t_{0}+\bar{\tau}}^{t} \mathbb{E}|y(s-2 \tau(s))|^{p} \mathrm{~d} s \\
& \leq 6^{p-1} k^{p} \frac{1}{1-\mu} \int_{t_{0}}^{t-\bar{\tau}} \mathbb{E}|y(s)|^{p} \mathrm{~d} s+6^{p-1} k^{p} \frac{1}{1-2 \mu} \int_{t_{0}-\bar{\tau}}^{t-2 \bar{\tau}} \mathbb{E}|y(s)|^{p} \mathrm{~d} s \\
& \leq 6^{p-1} k^{p} \frac{1}{1-\mu} \int_{t_{0}}^{t-\bar{\tau}} \mathbb{E}|y(s)|^{p} \mathrm{~d} s+6^{p-1} k^{p} \frac{1}{1-2 \mu} \int_{t_{0}-\bar{\tau}}^{t_{0}} \mathbb{E}|y(s)|^{p} \mathrm{~d} s \\
& +6^{p-1} k^{p} \frac{1}{1-2 \mu} \int_{t_{0}}^{t-\bar{\tau}} \mathbb{E}|y(s)|^{p} \mathrm{~d} s \\
& \leq 6^{p-1} k^{p} \frac{\bar{\tau}}{1-2 \mu}\left(\sup _{t_{0}-\bar{\tau} \leq s \leq t_{0}} \mathbb{E}|y(s)|^{p}\right)+6^{p-1} k^{p}\left(\frac{1}{1-\mu}+\frac{1}{1-2 \mu}\right) \int_{t_{0}}^{t-\bar{\tau}} \mathbb{E}|y(s)|^{p} \mathrm{~d} s \\
& \leq 6^{p-1} k^{p} \frac{\bar{\tau}}{1-2 \mu}\left(\sup _{t_{0}-\bar{\tau} \leq s \leq t_{0}} \mathbb{E}|y(s)|^{p}\right)+6^{p-1} k^{p}\left(\frac{1}{1-\mu}+\frac{1}{1-2 \mu}\right) \int_{t_{0}}^{t_{0}+\bar{\tau}} \mathbb{E}|y(s)|^{p} \mathrm{~d} s \\
& +6^{p-1} k^{p}\left(\frac{1}{1-\mu}+\frac{1}{1-2 \mu}\right) \int_{t_{0}+\bar{\tau}}^{t_{0}-\bar{\tau}+2 \Delta} \mathbb{E}|y(s)|^{p} \mathrm{~d} s \\
& \leq 6^{p-1} k^{p} \frac{\bar{\tau}}{1-2 \mu}\left(\sup _{t_{0}-\bar{\tau} \leq s \leq t_{0}} \mathbb{E}|y(s)|^{p}\right) \\
& +6^{p-1} k^{p} \bar{\tau}\left(\frac{1}{1-\mu}+\frac{1}{1-2 \mu}\right)\left(\sup _{t_{0} \leq s \leq t_{0}+\bar{\tau}} \mathbb{E}|y(s)|^{p}\right) \\
& +(2 \Delta-2 \bar{\tau}) 6^{p-1} k^{p}\left(\frac{1}{1-\mu}+\frac{1}{1-2 \mu}\right)\left(\sup _{t_{0}+\bar{\tau} \leq s \leq t_{0}-\bar{\tau}+2 \Delta} \mathbb{E}|y(s)|^{p}\right) \\
& \leq 6^{p-1} k^{p}\left[\frac{\bar{\tau}}{1-2 \mu}+\bar{\tau}\left(\frac{1}{1-\mu}+\frac{1}{1-2 \mu}\right)\right]\left(\sup _{t_{0}-\bar{\tau} \leq s \leq t_{0}+\bar{\tau}} \mathbb{E}|y(s)|^{p}\right) \\
& +2 \Delta 6^{p-1} k^{p}\left(\frac{1}{1-\mu}+\frac{1}{1-2 \mu}\right)\left(\sup _{t_{0}+\bar{\tau} \leq s \leq t_{0}-\bar{\tau}+2 \Delta} \mathbb{E}|y(s)|^{p}\right) \text {. }
\end{aligned}
$$


By reversing the order of integration, we get

$$
\begin{aligned}
& \int_{t_{0}+\bar{\tau}}^{t} \mathrm{~d} s \int_{s-\bar{\tau}}^{s} \mathbb{E}|y(r)|^{p} \mathrm{~d} r \\
& \quad=\int_{t_{0}}^{t} \mathrm{~d} r \int_{\max \left(t_{0}+\bar{\tau}, r\right)}^{\min (r+\bar{\tau}, t)} \mathbb{E}|y(s)|^{p} \mathrm{~d} s \\
& \quad \leq \bar{\tau} \int_{t_{0}}^{t} \mathbb{E}|y(s)|^{p} \mathrm{~d} s .
\end{aligned}
$$

Similarly, we can derive

$$
\begin{aligned}
& \int_{t_{0}+\bar{\tau}}^{t} \mathrm{~d} s \int_{s-\bar{\tau}}^{s} \mathbb{E}|y(r-\tau(r))|^{p} \mathrm{~d} r \\
& \quad=\int_{t_{0}}^{t} \mathrm{~d} r \int_{\max \left(t_{0}+\bar{\tau}, r\right)}^{\min (r+\bar{\tau}, t)} \mathbb{E}|y(s-\tau(s))|^{p} \mathrm{~d} s \\
& \quad \leq \bar{\tau} \int_{t_{0}}^{t} \mathbb{E}|y(s-\tau(s))|^{p} \mathrm{~d} s \\
& \quad \leq \frac{\bar{\tau}}{1-\mu} \int_{t_{0}-\bar{\tau}}^{t} \mathbb{E}|y(s)|^{p} \mathrm{~d} s \\
& \quad \leq \frac{\bar{\tau}^{2}}{1-\mu}\left(\sup _{t_{0}-\bar{\tau} \leq s \leq t_{0}} \mathbb{E}|y(s)|^{p}\right)+\frac{\bar{\tau}}{1-\mu} \int_{t_{0}}^{t} \mathbb{E}|y(s)|^{p} \mathrm{~d} s .
\end{aligned}
$$

Therefore, when $t \geq t_{0}+\bar{\tau}$, by substituting (3.4)-(3.6) into (3.3), we have

$$
\begin{aligned}
\int_{t_{0}+\bar{\tau}}^{t} \mathbb{E}|y(s)-y(s-\tau(s))|^{p} \mathrm{~d} s & { }^{p-1} k^{p} \bar{\tau}\left(\frac{1}{1-\mu}+\frac{2}{1-2 \mu}\right)\left(\sup _{t_{0}-\bar{\tau} \leq s \leq t_{0}+\bar{\tau}} \mathbb{E}|y(s)|^{p}\right) \\
+ & {\left[(6 \bar{\tau})^{p-1} K_{1}^{p}+\frac{1}{6 \bar{\tau}}[2 \bar{\tau} p(p-1)]^{p / 2}\left(3 K_{2}\right)^{p}\right] \frac{\bar{\tau}^{2}}{1-\mu}\left(\sup _{t_{0}-\bar{\tau} \leq s \leq t_{0}+\bar{\tau}} \mathbb{E}|y(s)|^{p}\right) } \\
& +2 \Delta 6^{p-1} k^{p}\left(\frac{1}{1-\mu}+\frac{1}{1-2 \mu}\right)\left(\sup _{t_{0}+\bar{\tau} \leq s \leq t_{0}-\bar{\tau}+2 \Delta} \mathbb{E}|y(s)|^{p}\right) \\
& +\left[(6 \bar{\tau})^{p-1} K_{1}^{p}+\frac{1}{6 \bar{\tau}}[2 \bar{\tau} p(p-1)]^{p / 2}\left(3 K_{2}\right)^{p}\right] \bar{\tau}\left(\frac{1}{1-\mu}+1\right) \int_{t_{0}}^{t} \mathbb{E}|y(r)|^{p} \mathrm{~d} r \\
:= & c_{2}\left(\sup _{t_{0}-\bar{\tau} \leq s \leq t_{0}+\bar{\tau}} \mathbb{E}|y(s)|^{p}\right)+c_{3} \int_{t_{0}}^{t} \mathbb{E}|y(r)|^{p} \mathrm{~d} r \\
& +2 \Delta 6^{p-1} k^{p}\left(\frac{1}{1-\mu}+\frac{1}{1-2 \mu}\right)\left(\sup _{t_{0}+\bar{\tau} \leq s \leq t_{0}-\bar{\tau}+2 \Delta} \mathbb{E}|y(s)|^{p}\right) .
\end{aligned}
$$

From (3.7) and (3.2), when $t \leq t_{0}+2 \Delta$, we get

$$
\begin{aligned}
& \mathbb{E}\left|x(t)-y(t)+G(y(t-\tau(t)))-G\left(y\left(t_{0}-\tau\left(t_{0}\right)\right)\right)\right|^{p} \\
& \quad \leq\left(2^{p-1}+1\right) c_{1} \int_{t_{0}}^{t} \mathbb{E}|x(s)-y(s)|^{p} \mathrm{~d} s+2^{p-1} c_{1} \int_{t_{0}}^{t_{0}+\bar{\tau}} \mathbb{E}|y(s)-y(s-\tau(s))|^{p} \mathrm{~d} s
\end{aligned}
$$




$$
\begin{aligned}
& +2^{p-1} c_{1} \int_{t_{0}+\bar{\tau}}^{t} \mathbb{E}|y(s)-y(s-\tau(s))|^{p} \mathrm{~d} s \\
\leq & \left(2^{p-1}+1\right) c_{1} \int_{t_{0}}^{t} \mathbb{E}|x(s)-y(s)|^{p} \mathrm{~d} s+4^{p-1} c_{1} \bar{\tau}\left(1+\frac{1}{1-\mu}\right)\left(\sup _{t_{0}-\bar{\tau} \leq s \leq t_{0}+\bar{\tau}} \mathbb{E}|y(s)|^{p}\right) \\
& +2^{p-1} c_{1} c_{2}\left(\sup _{t_{0}-\bar{\tau} \leq s \leq t_{0}+\bar{\tau}} \mathbb{E}|y(s)|^{p}\right)+2^{p-1} c_{1} c_{3} \int_{t_{0}}^{t} \mathbb{E}|y(s)-x(s)+x(s)|^{p} \mathrm{~d} s \\
& +2 c_{1} \Delta 12^{p-1} k^{p}\left(\frac{1}{1-\mu}+\frac{1}{1-2 \mu}\right)\left(\sup _{t_{0}+\bar{\tau} \leq s \leq t_{0}-\bar{\tau}+2 \Delta} \mathbb{E}|y(s)|^{p}\right) \\
\leq & \left(2^{p-1}+1\right) c_{1} \int_{t_{0}}^{t} \mathbb{E}|x(s)-y(s)|^{p} \mathrm{~d} s+4^{p-1} c_{1} \bar{\tau}\left(1+\frac{1}{1-\mu}\right)\left(\sup _{t_{0}-\bar{\tau} \leq s \leq t_{0}+\bar{\tau}} \mathbb{E}|y(s)|^{p}\right) \\
& +2^{p-1} c_{1} c_{2}\left(\sup _{t_{0}-\bar{\tau} \leq s \leq t_{0}+\bar{\tau}} \mathbb{E}|y(s)|^{p}\right)+4^{p-1} c_{1} c_{3} \int_{t_{0}}^{t} \mathbb{E}|x(s)-y(s)|^{p} \mathrm{~d} s \\
& +c_{1} \Delta 6^{p-1}(2 k)^{p}\left(\frac{1}{1-\mu}+\frac{1}{1-2 \mu}\right)\left(\sup _{t_{0}+\bar{\tau} \leq s \leq t_{0}-\bar{\tau}+2 \Delta} \mathbb{E}|y(s)|^{p}\right) \\
& +\frac{c_{1} c_{3}}{4 p \beta}(4 \alpha)^{p}\left(\sup _{t_{0}-\bar{\tau} \leq s \leq t_{0}} \mathbb{E}|y(s)|^{p}\right) \\
\leq & {\left[\left(2^{p-1}+1\right) c_{1}+4^{p-1} c_{1} c_{3}\right] \int_{t_{0}}^{t} \mathbb{E}|x(s)-y(s)|^{p} \mathrm{~d} s } \\
& +c_{1} \Delta 6^{p-1}(2 k)^{p}\left(\frac{1}{1-\mu}+\frac{1}{1-2 \mu}\right)\left(\sup _{t_{0}+\bar{\tau} \leq s \leq t_{0}-\bar{\tau}+2 \Delta} \mathbb{E}|y(s)|^{p}\right) \\
& +\left[2^{p-1} c_{1} c_{2}+\frac{c_{1} c_{3}}{4 p \beta}(4 \alpha)^{p}+4^{p-1} c_{1} \bar{\tau}\left(1+\frac{1}{1-\mu}\right)\right]\left(\sup _{t_{0}-\bar{\tau} \leq s \leq t_{0}+\bar{\tau}} \mathbb{E}|y(s)|^{p}\right) .
\end{aligned}
$$

Note that $\Delta \geq 2 \bar{\tau}$, when $t_{0}+\bar{\tau} \leq t \leq t_{0}+2 \Delta$, by Assumption 2.3, we have

$$
\begin{aligned}
\mathbb{E} \mid & G(y(t-\tau(t)))-\left.G\left(y\left(t_{0}-\tau\left(t_{0}\right)\right)\right)\right|^{p} \\
\leq & k^{p} \mathbb{E}\left|y(t-\tau(t))-y\left(t_{0}-\tau\left(t_{0}\right)\right)\right|^{p} \\
\leq & 2^{p-1} k^{p}\left(\sup _{t_{0}-\bar{\tau} \leq s \leq t_{0}+\bar{\tau}} \mathbb{E}|y(s)|^{p}\right)+2^{p-1} k^{p}\left(\sup _{t_{0}+\bar{\tau} \leq s \leq t_{0}+2 \Delta} \mathbb{E}|y(s-\tau(s))|^{p}\right) \\
\leq & 2^{p-1} k^{p}\left(\sup _{t_{0}-\bar{\tau} \leq s \leq t_{0}+\bar{\tau}} \mathbb{E}|y(s)|^{p}\right)+\frac{2^{p-1} k^{p}}{1-\mu}\left(\sup _{t_{0} \leq s \leq t_{0}-\bar{\tau}+2 \Delta} \mathbb{E}|y(s)|^{p}\right) \\
\leq & 2^{p-1} k^{p}\left(1+\frac{1}{1-\mu}\right)\left(\sup _{t_{0}-\bar{\tau} \leq s \leq t_{0}+\bar{\tau}} \mathbb{E}|y(s)|^{p}\right) \\
& \quad+\frac{2^{p-1} k^{p}}{1-\mu}\left(\sup _{t_{0}+\bar{\tau} \leq s \leq t_{0}-\bar{\tau}+2 \Delta} \mathbb{E}|y(s)|^{p}\right) .
\end{aligned}
$$

By (3.8) and (3.9), we further have

$$
\begin{aligned}
& \mathbb{E}|x(t)-y(t)|^{p} \\
& \quad \leq 2^{p-1} \mathbb{E}\left|x(t)-y(t)+G(y(t-\tau(t)))-G\left(y\left(t_{0}-\tau\left(t_{0}\right)\right)\right)\right|^{p} \\
& \quad+2^{p-1} \mathbb{E}\left|G(y(t-\tau(t)))-G\left(y\left(t_{0}-\tau\left(t_{0}\right)\right)\right)\right|^{p}
\end{aligned}
$$




$$
\begin{aligned}
\leq & {\left[\left(4^{p-1}+2^{p-1}\right) c_{1}+8^{p-1} c_{1} c_{3}\right] \int_{t_{0}}^{t} \mathbb{E}|x(s)-y(s)|^{p} \mathrm{~d} s } \\
& +c_{1} \Delta 12^{p-1}(2 k)^{p}\left(\frac{1}{1-\mu}+\frac{1}{1-2 \mu}\right)\left(\sup _{t_{0}+\bar{\tau} \leq s \leq t_{0}-\bar{\tau}+2 \Delta} \mathbb{E}|y(s)|^{p}\right) \\
& +\left[4^{p-1} c_{1} c_{2}+\frac{c_{1} c_{3}}{8 p \beta}(8 \alpha)^{p}+8^{p-1} c_{1} \bar{\tau}\left(1+\frac{1}{1-\mu}\right)\right]\left(\sup _{t_{0}-\bar{\tau} \leq s \leq t_{0}+\bar{\tau}} \mathbb{E}|y(s)|^{p}\right) \\
& +4^{p-1} k^{p}\left(1+\frac{1}{1-\mu}\right)\left(\sup _{t_{0}-\bar{\tau} \leq s \leq t_{0}+\bar{\tau}} \mathbb{E}|y(s)|^{p}\right) \\
& +\frac{4^{p-1} k^{p}}{1-\mu}\left(\sup _{t_{0}+\bar{\tau} \leq s \leq t_{0}-\bar{\tau}+2 \Delta} \mathbb{E}|y(s)|^{p}\right) \\
\leq & {\left[\left(4^{p-1}+2^{p-1}\right) c_{1}+8^{p-1} c_{1} c_{3}\right] \int_{t_{0}}^{t} \mathbb{E}|x(s)-y(s)|^{p} \mathrm{~d} s } \\
& +\left[c_{1} \Delta 12^{p-1}(2 k)^{p}\left(\frac{1}{1-\mu}+\frac{1}{1-2 \mu}\right)+\frac{4^{p-1} k^{p}}{1-\mu}\right]\left(\sup _{t_{0}+\bar{\tau} \leq s \leq t_{0}-\bar{\tau}+2 \Delta} \mathbb{E}|y(s)|^{p}\right) \\
& +\left[4^{p-1} c_{1} c_{2}+\frac{c_{1} c_{3}}{8 p \beta}(8 \alpha)^{p}+\left(8^{p-1} c_{1} \bar{\tau}+4^{p-1} k^{p}\right)\left(1+\frac{1}{1-\mu}\right)\right]\left(\sup _{t_{0}-\bar{\tau} \leq s \leq t_{0}+\bar{\tau}} \mathbb{E}|y(s)|^{p}\right) \\
:= & c_{4} \int_{t_{0}}^{t} \mathbb{E}|x(s)-y(s)|^{p} \mathrm{~d} s+c_{5}\left(\sup _{t_{0}+\bar{\tau} \leq s \leq t_{0}-\bar{\tau}+2 \Delta} \mathbb{E}|y(s)|^{p}\right)+c_{6}\left(\sup _{t_{0}-\bar{\tau} \leq s \leq t_{0}+\bar{\tau}} \mathbb{E}|y(s)|^{p}\right) \\
\leq & c_{4} \int_{t_{0}}^{t} \mathbb{E}|x(s)-y(s)|^{p} \mathrm{~d} s+c_{5}\left(\sup _{t_{0}-\bar{\tau}+\Delta \leq s \leq t_{0}-\bar{\tau}+2 \Delta} \mathbb{E}|y(s)|^{p}\right) \\
& +\left(c_{5}+c_{6}\right)\left(\sup _{t_{0}-\bar{\tau} \leq s \leq t_{0}-\bar{\tau}+\Delta} \mathbb{E}|y(s)|^{p}\right) .
\end{aligned}
$$

When $t_{0}+\bar{\tau} \leq t \leq t_{0}+2 \Delta$, by applying the Gronwall inequality [2], we get

$$
\begin{aligned}
\mathbb{E}|x(t)-y(t)|^{p} \leq & c_{5} \exp \left(2 \Delta c_{4}\right)\left(\sup _{t_{0}-\bar{\tau} \leq s \leq t_{0}-\bar{\tau}+\Delta} \mathbb{E}|y(s)|^{p}\right) \\
& +\left(c_{5}+c_{6}\right) \exp \left(2 \Delta c_{4}\right)\left(\sup _{t_{0}-\bar{\tau}+\Delta \leq s \leq t_{0}-\bar{\tau}+2 \Delta} \mathbb{E}|y(s)|^{p}\right) .
\end{aligned}
$$

Therefore,

$$
\begin{aligned}
\mathbb{E}|y(t)|^{p} \leq & {\left[2^{p-1}\left(c_{5}+c_{6}\right) \exp \left(2 \Delta c_{4}\right)+2^{p-1} \alpha^{p} \exp \left(-p \beta\left(t-t_{0}\right)\right)\right]\left(\sup _{t_{0}-\bar{\tau} \leq s \leq t_{0}-\bar{\tau}+\Delta} \mathbb{E}|y(s)|^{p}\right) } \\
& +2^{p-1} c_{5} \exp \left(2 \Delta c_{4}\right)\left(\sup _{t_{0}-\bar{\tau}+\Delta \leq s \leq t_{0}-\bar{\tau}+2 \Delta} \mathbb{E}|y(s)|^{p}\right),
\end{aligned}
$$

when $t_{0}-\bar{\tau}+\Delta \leq s \leq t_{0}-\bar{\tau}+2 \Delta$.

From (3.1), if $\bar{\tau}<\tilde{\tau}, 1-2^{p-1} c_{5} \exp \left(2 \Delta c_{4}\right)>0$, we have

$$
\begin{aligned}
\sup _{t_{0}-\bar{\tau}+\Delta \leq s \leq t_{0}-\bar{\tau}+2 \Delta} \mathbb{E}|y(s)|^{p} \leq & \frac{2^{p-1}\left(c_{5}+c_{6}\right) \exp \left(2 \Delta c_{4}\right)+2^{p-1} \alpha^{p} \exp \left(-p \beta\left(t-t_{0}\right)\right)}{1-2^{p-1} c_{5} \exp \left(2 \Delta c_{4}\right)} \\
& \times\left(\sup _{t_{0}-\bar{\tau} \leq s \leq t_{0}-\bar{\tau}+\Delta} \mathbb{E}|y(s)|^{p}\right),
\end{aligned}
$$

where $\hat{c}=\frac{2^{p-1}\left(c_{5}+c_{6}\right) \exp \left(2 \Delta c_{4}\right)+2^{p-1} \alpha^{p} \exp \left(-p \beta\left(t-t_{0}\right)\right)}{1-2^{p-1} c_{5} \exp \left(2 \Delta c_{4}\right)}$. Also, when $\bar{\tau}<\tilde{\tau}, \hat{c}<1$. 
When $t_{0}-\bar{\tau}+\Delta \leq s \leq t_{0}-\bar{\tau}+2 \Delta$, let

$$
\frac{\partial \ln \hat{c}}{\partial \varepsilon}=0
$$

We have

$$
\begin{aligned}
& {\left[\frac{\partial}{\partial \varepsilon}\left(c_{5}+c_{6}\right)+2 \Delta\left(c_{5}+c_{6}\right) \frac{\partial}{\partial \varepsilon} c_{4}\right]\left(1-2^{p-1} c_{5} \exp \left(2 \Delta c_{4}\right)\right)+\left[2^{p-1}\left(c_{5}+c_{6}\right) \exp \left(2 \Delta c_{4}\right)\right.} \\
& \left.+2^{p-1} \alpha^{p} \exp \left(-p \beta\left(t-t_{0}\right)\right)\right] \times\left[\frac{\partial}{\partial \varepsilon} c_{5}+2 \Delta c_{5} \frac{\partial}{\partial \varepsilon} c_{4}\right]=0
\end{aligned}
$$

that is,

$$
\begin{aligned}
& \left\{\left[\left(c_{51}+c_{61}\right)+2 \Delta c_{41} 4^{p-1} k^{p}\left(1+\frac{2}{1-\mu}\right)\right]\right. \\
& \quad+c_{1}\left[2 \Delta c_{41}\left(c_{51}+c_{61}\right)+\Delta c_{41} c_{51}(2 \alpha)^{p} \exp \left(-p \beta\left(t-t_{0}\right)\right)\right] \\
& \quad+\left[c_{51} 8^{p-1} k^{p}\left(1+\frac{2}{1-\mu}\right)-\left(c_{51}+c_{61}\right) \frac{8^{p-1} k^{p}}{1-\mu}\right] \exp \left(2 \Delta c_{41} c_{1}\right) \\
& \left.\quad+\left[2^{p-1} c_{51}+2 \Delta c_{41} \frac{8^{p-1} k^{p}}{1-\mu}\right] \alpha^{p} \exp \left(-p \beta\left(t-t_{0}\right)\right)\right\} \\
& \quad \times\left[(4 \Delta)^{p-1} K_{1}^{p}(1-p) \frac{1}{\varepsilon^{p}}+\frac{1}{4 \Delta}[4 \Delta p(p-1)]^{p / 2} K_{2}^{p}(1-p) \frac{-1}{(1-\varepsilon)^{p}}\right]=0
\end{aligned}
$$

where

$$
\begin{aligned}
& c_{12}=(4 \Delta)^{p-1} K_{1}^{p}, \quad c_{13}=\frac{1}{4 \Delta}[4 \Delta p(p-1)]^{p / 2} K_{2}^{p}, \\
& c_{41}=4^{p-1}+2^{p-1}+8^{p-1} c_{3}, \quad c_{51}=12^{p-1} \Delta(2 k)^{p}\left(\frac{1}{1-\mu}+\frac{1}{1-2 \mu}\right) \\
& c_{61}=4^{p-1} c_{2}+\frac{c_{3}}{8 p \beta}(8 \alpha)^{p}+8^{p-1} \bar{\tau}\left(1+\frac{1}{1-\mu}\right) .
\end{aligned}
$$

Thus, we further have

$$
\begin{aligned}
& {\left[(4 \Delta)^{p-1}\left(-K_{1}\right)^{p}-\frac{1}{4 \Delta}[4 \Delta p(p-1)]^{p / 2} K_{2}^{p}\right] \varepsilon^{p}} \\
& +(4 \Delta)^{p-1} K_{1}^{p}(-1)^{p-1} \varepsilon^{p-1}+\cdots+(4 \Delta)^{p-1} K_{1}^{p}=0 \text { or } \\
& {\left[\left(c_{51}+c_{61}\right)+2 \Delta c_{41} 4^{p-1} k^{p}\left(1+\frac{2}{1-\mu}\right)\right]} \\
& +\left[c_{51} 8^{p-1} k^{p}\left(1+\frac{2}{1-\mu}\right)-\left(c_{51}+c_{61}\right) \frac{8^{p-1} k^{p}}{1-\mu}\right] \\
& \quad \times \exp \left(2 \Delta c_{41} c_{1}\right)+\left[2^{p-1} c_{51}+2 \Delta c_{41} \frac{8^{p-1} k^{p}}{1-\mu}\right] \alpha^{p} \exp \left(-p \beta\left(t-t_{0}\right)\right) \\
& +\left[c_{12} \varepsilon^{1-p}+c_{13}(1-\varepsilon)^{1-p}\right] \\
& \quad \times\left[2 \Delta c_{41}\left(c_{51}+c_{61}\right)+\Delta c_{41} c_{51}(2 \alpha)^{p} \exp \left(-p \beta\left(t-t_{0}\right)\right)\right]=0 .
\end{aligned}
$$


From (3.16), it is easy to see that there exists a unique $\bar{\tau}$ such that $\bar{\tau}=\tau_{\max }$, when $\varepsilon \in$ $(0,1)$.

Choosing $\gamma=-\hat{c} / \Delta$, we have

$$
\sup _{t_{0}-\bar{\tau}+\Delta \leq t \leq t_{0}-\bar{\tau}+2 \Delta} \mathbb{E}\left|y\left(t ; t_{0} ; \psi\right)\right|^{p} \leq \exp (-\gamma \Delta)\left(\sup _{t_{0}-\bar{\tau} \leq t \leq t_{0}-\bar{\tau}+\Delta} \mathbb{E}\left|y\left(t ; t_{0} ; \psi\right)\right|^{p}\right) .
$$

Then, for any positive integer $m=1,2, \ldots$, by the existence and uniqueness of the state of system (2.1), when $t \geq t_{0}-\bar{\tau}+(m-1) \Delta$, we have

$$
y\left(t ; t_{0}, \psi\right)=y\left(t ; t_{0}-\bar{\tau}+(m-1) \Delta, \tilde{y}\left(t_{0}-\bar{\tau}+(m-1) \Delta ; t_{0}, \psi\right)\right) .
$$

From (3.17) and (3.18)

$$
\begin{aligned}
& \sup _{t_{0}-\bar{\tau}+m \Delta \leq t \leq t_{0}-\bar{\tau}+(m+1) \Delta} \mathbb{E}\left|y\left(t ; t_{0} ; \psi\right)\right|^{p} \\
&=\left(\sup _{t_{0}-\bar{\tau}+(m-1) \Delta+\Delta \leq t \leq t_{0}-\bar{\tau}+(m-1) \Delta+2 \Delta} \mathbb{E} \mid y\left(t ; t_{0}-\bar{\tau}+(m-1) \Delta,\right.\right. \\
&\left.\left.\tilde{y}\left(t_{0}-\bar{\tau}+(m-1) \Delta ; t_{0}, \psi\right)\right)\left.\right|^{p}\right) \\
& \leq \exp (-\gamma \Delta)\left(\sup _{t_{0}-\bar{\tau}+(m-1) \Delta \leq t \leq t_{0}-\bar{\tau}+m \Delta} \mathbb{E}\left|y\left(t ; t_{0} ; \psi\right)\right|^{p}\right) \\
& \quad \ldots \\
& \leq \exp (-\gamma m \Delta)\left(\sup _{t_{0}-\bar{\tau} \leq t \leq t_{0}-\bar{\tau}+\Delta} \mathbb{E}\left|y\left(t ; t_{0} ; \psi\right)\right|^{p}\right) \\
&= c \exp (-\gamma m \Delta),
\end{aligned}
$$

where $c=\sup _{t_{0}-\bar{\tau} \leq t \leq t_{0}-\bar{\tau}+\Delta} \mathbb{E}\left|y\left(t ; t_{0} ; \psi\right)\right|^{p}$. Thus, for any $t \geq t_{0}+\Delta$, there exists a positive integer $m$ such that $t_{0}-\bar{\tau}+m \Delta \leq t \leq t_{0}-\bar{\tau}+(m+1) \Delta$, we have

$$
\begin{aligned}
& \mathbb{E}\left|y\left(t ; t_{0} ; \psi\right)\right|^{p} \\
& \quad \leq c \exp \left(-\gamma t+\gamma t_{0}+\gamma \Delta\right) \\
& \quad=c \exp (\gamma \Delta) \exp \left(-\gamma\left(t-t_{0}\right)\right) .
\end{aligned}
$$

The inequality (3.20) is also true when $t_{0}-\bar{\tau} \leq t \leq t_{0}-\bar{\tau}+\Delta$. So system (2.1) is $p$ th moment globally exponentially stable. According to Lemma 2.1, system (2.1) is also almost surely globally exponentially stable.

If let $p=2, \varepsilon=\frac{1}{2}$ in Theorem 3.1, we obtain the following corollary.

Corollary 3.1 Let Assumptions 2.1-2.3 hold and system (2.2) is pth moment globally exponentially stable, then system (2.1) is mean square globally exponentially stable and also almost surely globally exponentially stable, if $\bar{\tau}<\tilde{\tau}, \tilde{\tau}$ is a unique positive solution of the transcendental equation

$$
\begin{aligned}
& {\left[\left[96 \Delta k^{2}\left(\frac{1}{1-\mu}+\frac{1}{1-2 \mu}\right)+4 \tilde{c}_{2}+4 \tilde{c}_{3} \alpha^{2} / \beta+8 \bar{\tau}\left(1+\frac{1}{1-\mu}\right)\right] \tilde{c}_{1}+4 k^{2}\left(1+\frac{3}{1-\mu}\right)\right]} \\
& \quad \times 2 \exp \left(2 \Delta \tilde{c}_{1}\left(6+8 \tilde{c}_{3}\right)\right)+2 \alpha^{2} \exp (-2 \beta(\Delta-\bar{\tau}))=1
\end{aligned}
$$


where

$$
\begin{aligned}
& \tilde{c}_{1}=8 \Delta K_{1}^{2}+4 K_{2}^{2}, \\
& \tilde{c}_{2}=6 k^{2} \bar{\tau}\left(\frac{1}{1-\mu}+\frac{2}{1-2 \mu}\right)+\left(6 \bar{\tau} K_{1}^{2}+6 K_{2}^{2}\right) \frac{\bar{\tau}^{2}}{1-\mu}, \\
& \tilde{c}_{3}=\left(6 \bar{\tau} K_{1}^{2}+6 K_{2}^{2}\right) \bar{\tau}\left(1+\frac{1}{1-\mu}\right),
\end{aligned}
$$

$\Delta$ is a step, $\Delta-\bar{\tau}>\ln \left(2 \alpha^{2}\right) /(2 \beta)>0$.

Remark 3.1 Theorem 3.1 shows that when system (2.2) without delays and neutral terms is globally exponentially stable, system (2.1) induced by time delays and neutral terms can be globally exponentially stable and almost surely globally exponentially stable if the delays are smaller than the given upper bound and the neutral terms are sufficiently small.

Remark 3.2 From the proof of Theorem 3.1, we can see that the upper bound of parameter uncertainty delays is derived through a subtle inequality and a transcendental equation. By using software such as MATLAB or Mathematica, the derived conditions in the theorem can be verified easily.

\section{Numerical example}

In this section, we give an example with numerical simulation to illustrate our result in the preceding section.

Example 4.1 Consider a two-state neutral type delay system

$$
\left\{\begin{array}{l}
\mathrm{d}\left[y_{1}(t)-k \cos y_{2}(t-\tau(t))\right]=-0.06 y_{1}(t-\tau(t)) \mathrm{d} t+0.1 y_{2}(t-\tau(t)) \mathrm{d} w(t), \\
\mathrm{d}\left[y_{2}(t)-k \cos y_{1}(t-\tau(t))\right]=-0.06 y_{2}(t-\tau(t)) \mathrm{d} t+0.1 y_{1}(t-\tau(t)) \mathrm{d} w(t),
\end{array}\right.
$$

where $\tau(t)$ is the time-varying delay and $k \in(0,1)$. According to Theorem 4.4 in [2], the system without time-varying delays and neutral terms

$$
\left\{\begin{array}{l}
\mathrm{d} x_{1}(t)=-0.06 x_{1}(t) \mathrm{d} t+0.1 x_{2}(t) \mathrm{d} w(t) \\
\mathrm{d} x_{2}(t)=-0.06 x_{2}(t) \mathrm{d} t+0.1 x_{1}(t) \mathrm{d} w(t)
\end{array}\right.
$$

is globally exponentially stable with $\alpha=0.8, \beta=0.5$.

Let $\Delta=0.5, \mu=0, k=0.04, K_{1}=0.06, K_{2}=0.1$. By Corollary 3.1, choosing $\varepsilon=\frac{1}{2}$, and solving the following transcendental equation:

$$
\begin{aligned}
& {\left[0.054\left(0.1536+16.1152 \bar{\tau}+(0.864 \bar{\tau}+0.24) \bar{\tau}^{2}+10.24 \bar{\tau}(0.0216 \bar{\tau}+0.06)\right)+0.0256\right]} \\
& \quad \times 2 \exp (0.0544(6+16 \bar{\tau}(0.0216 \bar{\tau}+0.06)))+1.28 \exp (-0.5+\bar{\tau})=1
\end{aligned}
$$

we can obtain its unique solution $\tilde{\tau} \approx 0.2390$ and $\Delta-\tilde{\tau}=0.2610>\ln (1.28)=0.2469$. Thus, system (4.1) is globally exponentially stable (see Figure 1 , when $\tilde{\tau}=0.2390, k=0.04$ ). 


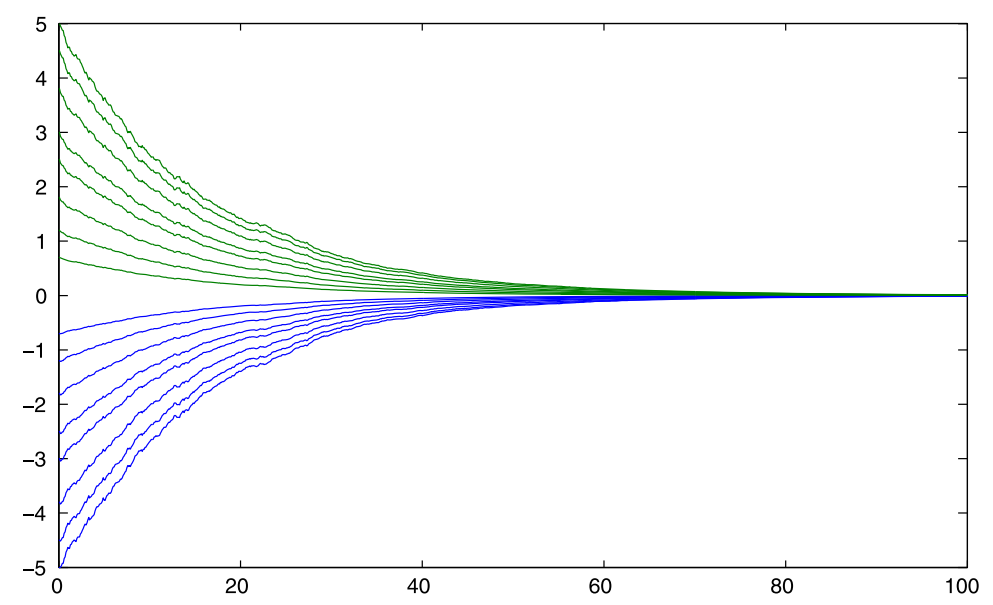

Figure 1 Stable states of the system in (4.1) with $\tilde{\tau}=0.2390, k=0.04$.

\section{Conclusion}

This paper studies the robust stability of global exponential stability of nonlinear stochastic systems in the presence of time-varying delays and neutral terms. The result shows that a globally exponentially stable nonlinear stochastic system perturbed by both time delays and neutral terms can sustain global exponential stability provided that the delays are, respectively, smaller than the given upper bounds and the neutral terms are sufficiently small.

\section{Competing interests}

The authors declare that they have no competing interests.

\section{Authors' contributions}

The authors declare that the study was realized in collaboration with the same responsibility. All of the authors read and approved the final version of the manuscript.

\section{Author details}

${ }^{1}$ College of Mathematics and Statistics, South Central University for Nationalities, Wuhan, 430074, P.R. China.

${ }^{2}$ Department of Mathematical Sciences, Loughborough University, Loughborough, Leicestershire LE11 3TU, UK. ${ }^{3}$ College of Science, Huazhong Agriculture University, Wuhan, 430070, P.R. China.

\section{Acknowledgements}

The authors would like to thank the referee and the associate editor for their very useful comments and suggestions. The authors would also like to thank the financial supports from the National Natural Science Foundation of China (Nos. 61374085, 61304068), Graduate Innovation Fund Projects of the South-Central University for Nationalities under Grant 2014sycxjj124 and Huazhong Agricultural University Scientific \& Technological Self-Innovation Foundation under Grant 2662014BQ059.

Received: 21 September 2014 Accepted: 7 January 2015 Published online: 31 March 2015

\section{References}

1. Kolmanovskii, V, Nosov, V: Stability and Periodic Modes of Control Systems with Aftereffect. Nauka, Moscow (1981)

2. Mao, X: Stochastic Differential Equations and Applications, 2nd edn. Horwood, Chichester (2007)

3. Kolmanovskii, V, Koroleva, N, Maizenberg, T, Mao, X, Matasov, A: Neutral stochastic differential delay equations with Markovian switching. Stoch. Anal. Appl. 21, 839-867 (2003)

4. Mao, X, Shen, Y, Yuan, C: Almost surely asymptotic stability of neutral stochastic differential delay equations with Markovian switching. Stoch. Process. Appl. 118, 1385-1406 (2007)

5. Bao, J, Hou, Z, Yuan, C: Stability in distribution of neutral stochastic differential delay equations with Markovian switching. Stat. Probab. Lett. 79, 1663-1673 (2009)

6. Liu, Y, Wang, Z, Liu, X: Global exponential stability of generalized recurrent neural networks with discrete and distributed delays. Neural Netw. 19,667-675 (2006)

7. Mao, X: Stability and stabilization of stochastic differential delay equations. IET Control Theory Appl. 1, 1551-1566 (2007) 
8. Sipahi, R, Niculescu, S, Abdallah, C, Michiels, W, Gu, K: Stability and stabilization of systems with time delay: limitations and opportunities. IEEE Control Syst. Mag. 38, 38-65 (2011)

9. Shen, $Y$, Wang, J: Noise-induced stabilization of the recurrent neural networks with mixed time-varying delays and Markovian-switching parameters. IEEE Trans. Neural Netw. 18, 1857-1862 (2007)

10. Shen, Y, Wang, J: Robustness analysis of global exponential stability of recurrent neural networks in the presence of time delays and random disturbances. IEEE Trans. Neural Netw. Learn. Syst. 23, 87-96 (2012)

11. Jiang, $F$, Yang, $H$, Shen, Y: On the robustness of global exponential stability for hybrid neural networks with noise and delay perturbations. Neural Comput. Appl. 24, 1497-1504 (2014)

12. Zhu, S, Luo, W, Shen, Y: Robustness analysis for connection weight matrices of global exponential stability of stochastic recurrent neural networks. Circuits Syst. Signal Process. 33, 2065-2083 (2014)

13. Zhu, S, Shen, Y: Robustness analysis of global exponential stability of neural networks with Markovian switching in the presence of time-varying delays or noises. Neural Comput. Appl. 23, 1563-1571 (2013)

14. Luo, W, Zhong, K, Zhu, S, Shen, Y: Further results on robustness analysis of global exponential stability of recurrent neural networks with time delays and random disturbances. Neural Netw. 53, 127-133 (2014)

15. Shen, Y, Wang, J: Robustness analysis of global exponential stability of non-linear systems with time delays and neutral terms. IET Control Theory Appl. 7, 1227-1232 (2013)

\section{Submit your manuscript to a SpringerOpen ${ }^{\circ}$ journal and benefit from:}

- Convenient online submission

Rigorous peer review

- Immediate publication on acceptance

- Open access: articles freely available online

- High visibility within the field

- Retaining the copyright to your article 